\title{
Estudo comparativo de controladores de Mamdani e Sugeno para controle de tráfego em interseções isoladas
}

\author{
Michelle Andrade ${ }^{1}$; Maria Alice Prudêncio Jacques ${ }^{2}$
}

\begin{abstract}
Resumo: Na definição de um controlador fuzzy que modele o conhecimento do especialista e promova o controle desejado de um determinado sistema, o projetista precisa selecionar, inicialmente, o tipo básico de controlador a ser projetado. Isto é, precisa definir se o controlador será do tipo proposto por Mamdani ou Sugeno. Na literatura são encontrados controladores semafóricos fuzzy dos dois tipos sem que, no entanto, referência seja feita às razões que levaram à adoção de um tipo em detrimento do outro. Visando cobrir essa lacuna, este trabalho analisa as principais características dos dois tipos de controladores, e compara as respostas e os efeitos dos mesmos no controle de diferentes volumes de tráfego em uma interseção isolada. Os resultados mostram que o controlador de Sugeno apresenta nítidas vantagens sobre o de Mamdani para a aplicação considerada.
\end{abstract}

Abstract: In defining the fuzzy controller that models specialist knowledge and promotes the desired control of a specific system, the planner needs to select firstly the basic type of controller to be projected. In other words, it is necessary to define if the controller will be the type proposed by Mamdani or Sugeno. In the literature, there are fuzzy traffic controllers from the two types without there being any explanation as to the choice of one over the other. With a view to filling this loophole, this study analyses the main characteristics of both controller types, and it compares their answers and results in controlling different traffic volumes at an isolated intersection. Results indicate that the Sugeno controller presents clear advantages vis a vis Madami’s in the application considered.

\section{INTRODUÇÃO}

Em 1973 Zadeh publicou um artigo que lançou as bases para a utilização da lógica fuzzy na análise de sistemas e processos de decisão cuja complexidade, segundo o autor, não permitia a utilização de técnicas matemáticas convencionais. Nesse trabalho foi cunhado o termo algoritmo fuzzy, definido como "um conjunto ordenado de instruções fuzzy cuja execução produz uma solução aproximada para um determinado problema” (Zadeh, 1973). De acordo com Kikuchi (1991), o emprego de algoritmos fuzzy para a abordagem e solução de problemas na área de transportes se justifica em função do grau de incerteza associado a vários desses problemas, como é o caso do controle do tráfego em interseções semaforizadas.

A utilização de algoritmos fuzzy na solução de problemas de controle foi iniciada com o trabalho pioneiro de Mamdani (1973, apud Sugeno, 1985), motivado pelos trabalhos de Zadeh. No controlador desenvolvido foi adotado um método para o processo de decisão baseado em regras do tipo "SE A ENTÃO B", nas quais tanto o antecedente quanto o conseqüente são valores de variáveis lingüísticas, expressos por meio

\footnotetext{
${ }^{1}$ Michelle Andrade, Departamento de Engenharia Civil e Ambiental, Faculdade de Tecnologia, Universidade de Brasília. Brasília, DF, Brasil (e-mail: maccivil@gmail.com).

${ }^{2}$ Maria Alice Prudêncio Jacques, Departamento de Engenharia Civil e Ambiental, Faculdade de Tecnologia, Universidade de Brasília. Brasília, DF, Brasil (e-mail: mapj@unb.br).
}

Manuscrito recebido em 8/7/2008 e aprovado para publicação em 9/10/2008. Este artigo é parte de TRANSPORTES, volume XVI, número 2, de dezembro de 2008. ISSN: 1415-7713. de conjuntos fuzzy (Mamdani e Assilian, 1975). Identificando algumas dificuldades para a utilização do processo de decisão adotado no controlador de Mamdani, Takagi e Sugeno (1983) propuseram um método de tomada de decisão simplificado, baseado na lógica fuzzy, onde somente o antecedente das regras é formado por variáveis fuzzy. O conseqüente de cada regra é expresso por uma função linear dos valores observados das variáveis que descrevem o estado do sistema (variáveis de entrada). Este tipo de controlador é referido na literatura como controlador de Sugeno (Lee, 1990a; Zimmermann, 1996; MathWorks, 2008).

Os controladores de Mamdani e Sugeno sofreram aprimoramentos ao longo do tempo e têm sido utilizados para diferentes aplicações (Lee, 1990a). Atualmente estão disponíveis em pacotes computacionais, com diferentes recursos para implementação dos seus componentes, o que facilita o desenvolvimento de controladores fuzzy para a solução de diferentes problemas de controle a partir da seleção de um desses tipos básicos. A escolha do tipo de controlador a adotar e, na seqüência, a forma de implementação dos seus diferentes componentes, exige do projetista um conhecimento geral dos aspectos teóricos envolvidos em cada solução possível e, além disso, uma prévia noção do impacto de cada escolha (tipo de controlador e implementação dos componentes) sobre a resposta do controlador e sobre a eficácia do controle por ele produzido.

No caso dos controladores semafóricos fuzzy, cujo primeiro modelo foi desenvolvido por Pappis e Mamdani (1977), a literatura mostra que a maioria dos tra- 
balhos adotou o controlador tipo Mamdani, pelo menos em termos conceituais gerais, especificando as saídas das regras como conjuntos fuzzy (Jacques et al., 2002a). Enquanto análises sobre o impacto das diferentes possibilidades de implementação de alguns elementos do controlador semafórico fuzzy podem ser encontradas na literatura (Jacques et al., 2002b, 2002c e 2003), estudos voltados à avaliação do impacto da escolha do tipo básico do controlador no desenvolvimento de controladores semafóricos fuzzy não encontram-se disponíveis.

O objetivo do presente trabalho é dar continuidade aos estudos realizados no âmbito da Universidade de Brasília e da Universidade de Tecnologia de Helsinki para o desenvolvimento de controladores semafóricos fuzzy para interseções isoladas, por meio da investigação do efeito provocado pela escolha do tipo do controlador (Mamdani ou Sugeno) sobre a resposta do dispositivo às solicitações do tráfego e sobre o desempenho do fluxo de tráfego. Apresenta, inicialmente, uma descrição dos controladores de Mamdani e de Sugeno, expondo suas diferenças conceituais e indicando como eles podem ser implementados com o auxilio de um programa computacional que disponha de recursos específicos para este fim, como é o caso do programa MATLAB ${ }^{\circledR}$ (MathWorks, 2008). Na seqüência, o estudo de caso realizado para investigar o impacto dos dois tipos de controladores é descrito. Foi analisada a operação de uma interseção isolada formada por duas vias de sentido único de circulação, uma via principal e outra secundária, sob três níveis diferentes de volume de tráfego. A análise é efetuada a partir dos resultados da simulação da operação do tráfego, realizada com o uso do programa HUTSIM (Kosonem, 1999).

Os estudos relacionados ao emprego de controladores semafóricos fuzzy para o controle de interseções isoladas, tanto com base em simulações da operação do tráfego quanto em alguns testes de campo, têm apresentado resultados promissores (Niittymäki e Nevala, 2001). Assim, contribuições ao aperfeiçoamento do processo de desenvolvimento desses controladores, como a descrita no presente trabalho, se revestem de importância no contexto atual da demanda dos gestores de tráfego por dispositivos de controle cada vez mais eficazes.

\section{TIPOS DE CONTROLADORES FUZZY}

Os controladores fuzzy descritos na literatura são classificados em função das características gerais do seu método de tomada de decisão. Embora diferentes métodos sejam apresentados na literatura (ver Lee, 1990b), de acordo com Sugeno (1985) eles podem ser reunidos em dois grandes grupos. O primeiro reúne os que são baseados nas funções de implicação fuzzy e em operadores de composição para a definição da saída fuzzy do controlador. Já os do segundo grupo dispensam a definição de funções de implicação e operadores para a inferência. Os controladores do tipo Mamdani, referidos nesta seção como controlador de Mamdani, são baseados no primeiro grupo, e os controladores do tipo desenvolvido por Takagi e Sugeno, aqui referidos como controlador de Sugeno, fazem parte do segundo grupo.

Nos dois tipos de controladores a ação de controle é obtida por meio da definição de um conjunto de instruções (ou regras) de controle fuzzy, isto é de um algoritmo fuzzy. Essas regras são do tipo GMP (Generalized Modus Ponens), e uma dada regra (Ri) pode ser apresentada como:

Antecedente: $x$ é $A^{\prime}$ e $y$ é $B$ '

Regra $\left(R_{i}\right)$ : se $x$ é $A_{i}$ e $y$ é $B_{i}$ então $z$ é $C_{i}$ Conseqüente: $z$ é $C^{\prime}{ }_{i}$

onde $x$ and $y$ são variáveis lingüísticas relacionadas ao estado do processo e z é a variável lingüística de controle; $A^{\prime}, A_{i}, B^{\prime}, B_{i}, C^{\prime}{ }_{i}$ e $C i$ são conjuntos fuzzy de $x, y$ e $z$ nos universos de discurso $U, V$ e $W$, respectivamente. Como será apresentado a seguir, no caso do controlador de Sugeno, o conseqüente da regra não é um conjunto fuzzy.

\subsection{Controlador de Mamdani}

Este controlador tem como base o trabalho pioneiro de Mamdani, publicado em 1973 (Mamdani, 1973). No algoritmo fuzzy do controlador, cada regra é uma proposição condicional fuzzy, e diferentes relações fuzzy em $U \times V \times W$ podem ser dela derivadas.

A implementação de cada regra é feita mediante a definição de operadores para o processamento do antecedente da regra e da função de implicação que irá definir o seu conseqüente. A ação do controlador fuzzy é definida pela agregação das $n$ regras $R_{i}$ que compõem o algoritmo, mediante o uso do conectivo "também”, o qual pode ser implementado por diferentes operadores. Esta agregação resulta no conjunto fuzzy $C$, que define a saída do controlador $C$. A saída efetiva do controlador é então obtida por meio de um processo de defuzificação aplicado ao conjunto $C$.

As diferentes possibilidades para a implementação dos conectores das regras, das funções de implicação e do processo de defuzificação são amplamente discutidas na literatura (ver, por exemplo, Lee 1990a e 1990b) e não serão tratadas no presente trabalho. O impacto da adoção de algumas dessas possibilidades, e da definição dos conjuntos fuzzy associados às variáveis de entrada do controlador semafórico fuzzy, foram objeto de pesquisas anteriores (Jacques et al., 
2002b, 2002c, 2003; Vaz, 2006).

\subsection{Controlador de Sugeno}

O controlador de Sugeno (Takagi e Sugeno, 1983) consiste numa simplificação do controlador de Mamdani, onde o conseqüente de cada regra é definido como uma função das variáveis lingüísticas de entrada. Isto é, a regra geral $R_{i}$ pode ser escrita como:

$$
\text { Regra }\left(R_{i}\right) \text { : se } x \text { é } A_{i} \text { e } y \text { é } B_{i} \text { então } z=f_{i}(x, y)
$$

O resultado de cada regra é, portanto, um valor numérico (não um conjunto fuzzy), que assume como peso o valor da pertinência resultante do processamento do antecedente da regra. Essa determinação dispensa, portanto, a definição de uma função de implicação específica. A resposta final do controlador é obtida pela média ponderada das respostas das regras individuais. Isto é, neste tipo de controlador não cabe processo de defuzificação.

$O$ valor de $z$ pode também ser definido como um valor constante, que pode ser interpretado como um conjunto fuzzy com a característica especial de apresentar um único valor com pertinência igual a um e todos os demais com pertinência zero. Este tipo de conjunto fuzzy é denominado singleton, e o seu emprego permite a definição de regras com valores de saída que representam uma classificação da resposta do controlador, sem alterar a forma simplificada da determinação da resposta final do controlador.

\subsection{Implementação dos controladores em programa computacional}

A implementação de um controlador fuzzy, para fins de utilização efetiva, requer o uso de programas computacionais. Atualmente, alguns programas de uso geral dispõem de módulos específicos para facilitar a realização desta tarefa, como é o caso do Fuzzy Logic Toolbox do MATLAB ${ }^{\circledR}$, usado neste trabalho para implementar os dois tipos de controladores em estudo.

O programa permite ao usuário definir os quatro componentes principais do sistema de inferência fuzzy, que são: fuzificação dos valores das variáveis de entrada e aplicação dos operadores que podem estar presentes no antecedente das regras ("e” e "ou”); implicação do antecedente sobre o conseqüente (função de implicação); agregação dos conseqüentes de todas as regras definidas; e a defuzificação do conjunto $\mathbf{C}$ de resposta. No MATLAB ${ }^{\circledR}$, antes de iniciar a definição dos componentes do sistema, o usuário deve indicar se o seu controlador é do tipo Mamadani ou Sugeno. Dependendo do tipo selecionado, são liberados os campos pertinentes para a entrada dos dados. Isto é, se for selecionado o controlador de Sugeno, por exemplo, os campos referentes à função de implicação, ao termo de agregação "também” e ao método de defuzificação ficam desabilitados. O programa apresenta diferentes opções ao usuário para a configuração dos componentes do sistema e, para a maioria dos campos, também permite a definição de outras alternativas.

\section{ESTUDO DE CASO}

A avaliação do desempenho dos controladores de Mamdani e Sugeno foi realizada a partir da implementação de dois controladores de cada tipo, definidos com base em elementos básicos comuns. O funcionamento dos controladores foi então testado para o controle de uma interseção isolada formada por duas vias de sentido único, uma principal e outra secundária. Este teste foi realizado através da simulação da operação do tráfego no local, com o auxílio do programa HUTSIM (Kosonen, 1999).

\subsection{Descrição das características gerais do controlador semafórico fuzzy}

O controlador básico, que foi implementado posteriormente como controlador de Mamdani e Sugeno, possui as características básicas a seguir descritas.

\subsubsection{Variáveis de entrada e saída do controlador}

As variáveis que descrevem o estado do sistema a ser controlado (variáveis de entrada) são "Fila" (na aproximação que está recendo indicação vermelha) e "Chegada” (número de veículos presentes nos 100 m próximos à linha de retenção da aproximação que está recebendo sinal verde). A variável "Fila” pode assumir os valores "pequena”, "média”, "longa” e "qualquer", que são definidos como conjuntos fuzzy. No caso da variável "Chegada”, os valores possíveis são “zero”, “pouca”, “média”, “muita” e "qualquer”. Os conjuntos fuzzy associados a estas variáveis, mostrados na Figura 1, são os mesmos definidos no estudo de Santos (2003), mais especificamente, o Caso 4 definido naquele trabalho. Dentre os casos estudados por Santos (2003) o Caso 4 apresentou os melhores resultados nas simulações realizadas em termos de atrasos e paradas veiculares. Estes conjuntos são derivados do controlador básico utilizado no trabalho de Andrade e Jacques (2005), definidos a partir do estudo de Niittymäki e Pursula (2000). Já a variável que descreve a saída do controlador é denominada "Extensão", e pode assumir os valores "zero", "curta”, "média” e "longa”. Os conjuntos fuzzy que representam esses valores são definidos de forma diferente para os dois tipos controladores e serão posteriormente apresentados. 


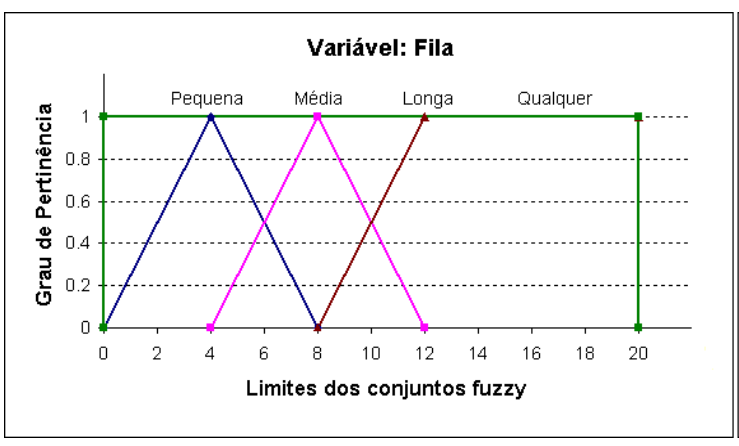

(a) Conjuntos fuzzy da variável "Fila"

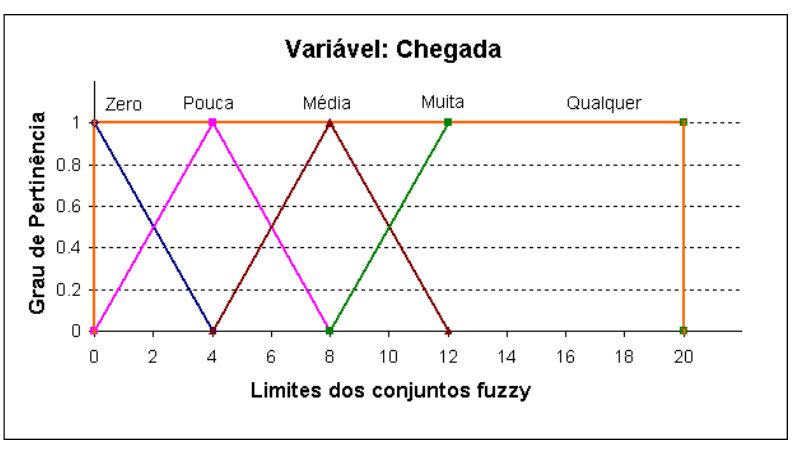

(b) Conjuntos fuzzy da variável "Chegada"

Figura 1: Conjuntos fuzzy de entrada do controlador básico

\subsubsection{Base de regras}

O conjunto de regras utilizado para o desenvolvimento dos dois controladores é o apresentado na Tabela 1, formado por 14 regras. Esta base de regras também foi empregada no trabalho de Andrade e Jacques (2005).

Tabela 1: Base de regras adotada

\begin{tabular}{lccc}
\hline Regra & Se $\boldsymbol{a}$ “Fila” & $\boldsymbol{e} \boldsymbol{a}$ “Chegada” & Então $\boldsymbol{a}$ “Extensão” é \\
\hline 1 & Qualquer & Zero & Zero \\
2 & Pequena & Pouca & Curta \\
3 & Média & Pouca & Zero \\
4 & Longa & Pouca & Zero \\
5 & Pequena & Média & Média \\
6 & Média & Média & Curta \\
7 & Longa & Média & Curta \\
8 & Pequena & Muita & Longa \\
9 & Média & Muita & Média \\
10 & Longa & Muita & Média \\
11 & - & Pouca & Curta \\
12 & - & Média & Média \\
13 & - & Muita & Longa \\
14 & Longa & Qualquer & Zero \\
\hline
\end{tabular}

Para a implementação do antecedente das regras nos dois controladores foi utilizado o operador "mínimo" para o conectivo "e".

\subsubsection{Condições gerais da operação}

Na operação do controlador simulada no programa HUTSIM, os seguintes procedimentos são adotados:

a) tempo mínimo de verde igual a 5 segundos para as duas aproximações;

b) máximo de 5 (cinco) extensões sucessivas de verde para cada aproximação;

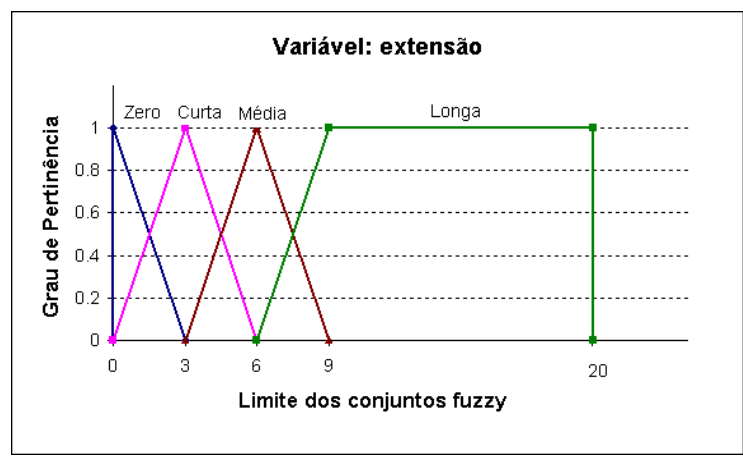

$\begin{array}{ll}\text { (a) Controlador } 1 & \text { (b) Controlador } 3\end{array}$

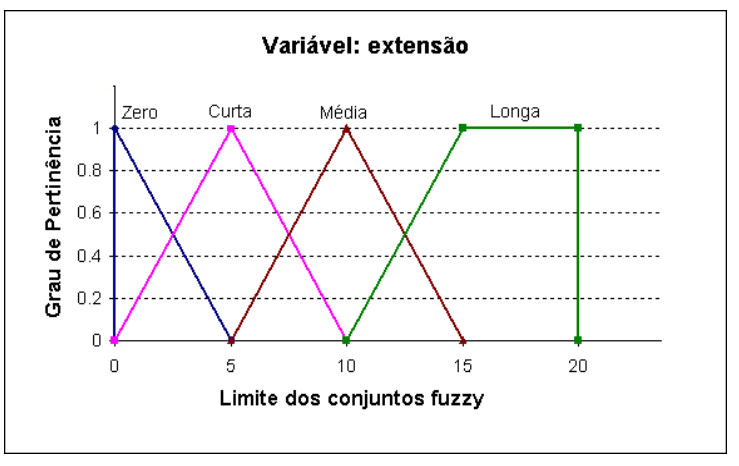

(b) Controlador 3

\subsection{Descrição do controlador do tipo Mamdani}

Os controladores de Mamdani testados (Controladores 1 e 3), além dos aspectos básicos já descritos, tiveram os valores de suas variáveis de saída definidos pelos conjuntos fuzzy mostrados na Figura 2.

A função de implicação adotada foi a de Mamdani, implementada com o operador “mínimo", e o método de defuzificação adotado foi o método do centro de gravidade, que assegura uma superfície de controle suave e contínua. Na Figura 3 são apresentadas, a título de ilustração, a base de regras implementada no MATLAB $^{\circledR}$ e a superfície de resposta correspondentes ao Controlador 3.

Os Controladores 1 e 3 apresentam como principal diferença o comprimento das suas extensões de tempo de verde, fruto da partição diferenciada do universo de discurso da variável extensão, na definição dos conjuntos fuzzy a ela associados em cada controlador (ver Figura 2). O Controlador 3 responde com extensões maiores, especialmente para chegadas veiculares pequenas.

Figura 2: Conjuntos fuzzy da variável de saída do controlador de Mamdani 


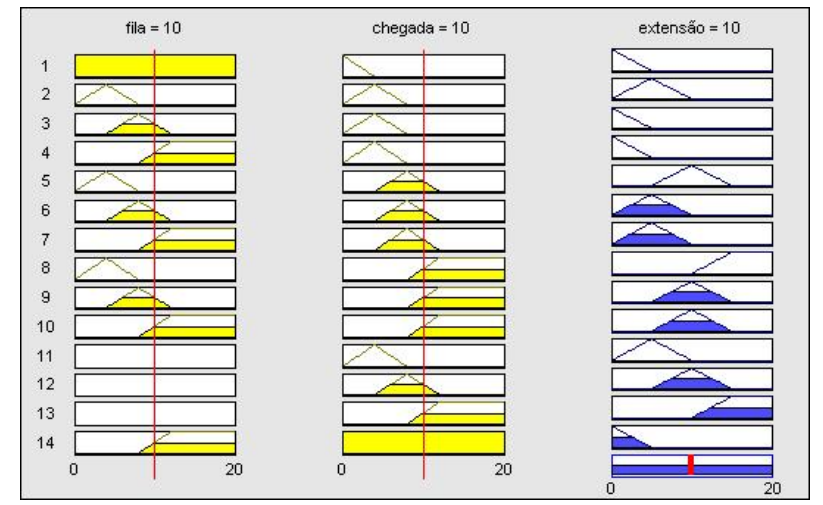

(a) Regras

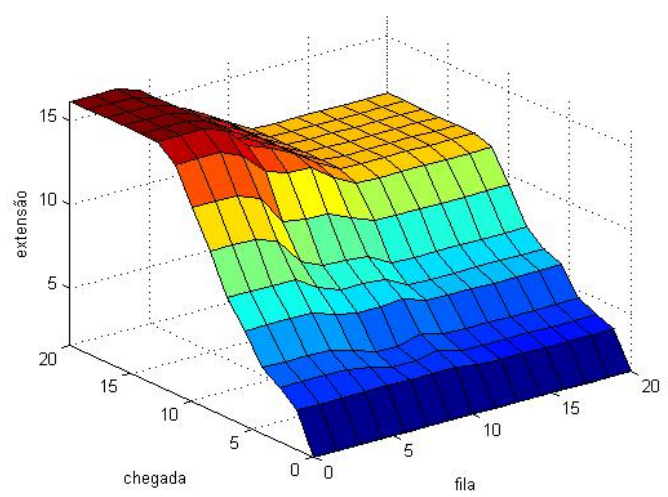

(b) Superfície de controle

Figura 3: Base de regras e Superfície de resposta do Controlador 3

\subsection{Descrição do controlador do tipo Sugeno}

Para efeito da descrição do controlador de Sugeno, cabe apenas acrescentar que, em conjunto com as características descritas na seção 3.1, a variável de saída foi definida com base nos conjuntos singleton mostrados na Figura 4.

Os conjuntos singletons da variável extensão foram definidos a partir dos pontos de máximo da mesma variável dos controladores do tipo Mamdani (controladores 1 e 3), os quais já foram utilizados em estudos anteriores tais como o de Bingham (1998) e de Andrade e Jacques (2005). Assim, os Controladores 2 e 4 também diferem entre si somente quanto aos conjuntos fuzzy da variável extensão, cujos valores com per- tinência 1 são superiores no Controlador 4, na ordem de $66 \%$.

A base de regras e a respectiva superfície de resposta do Controlador 4, implantado no MATLAB ${ }^{\circledR}$, são apresentadas na Figura 5 a título de exemplo do funcionamento do controlador de Sugeno.

\subsection{Características da situação de controle considerada}

Conforme já mencionado nos itens anteriores, o controle é realizado sobre uma interseção isolada, com duas vias de aproximação perpendiculares, compostas de duas faixas de trânsito de sentido único. A velocidade operacional da via é de $60 \mathrm{~km} / \mathrm{h}$ e o tráfego é composto apenas de carros de passeio.

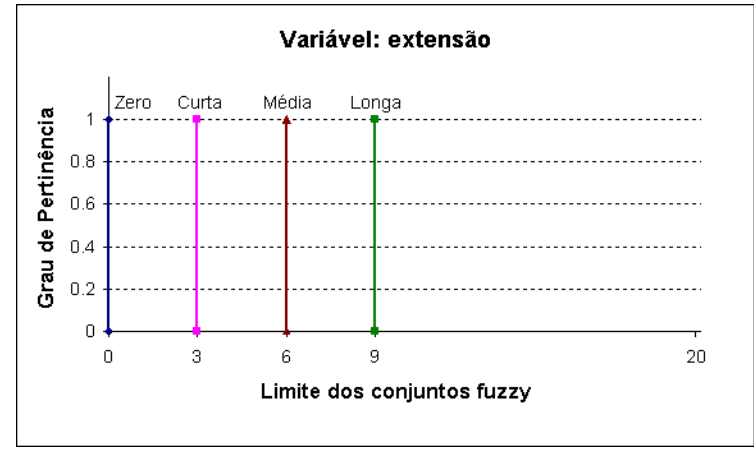

(a) Controlador 2

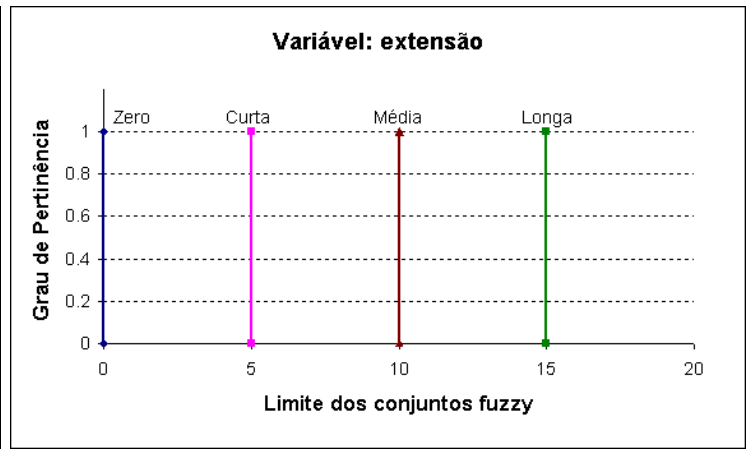

(b) Controlador 4

Figura 4: Valores da variável de saída do controlador de Sugeno

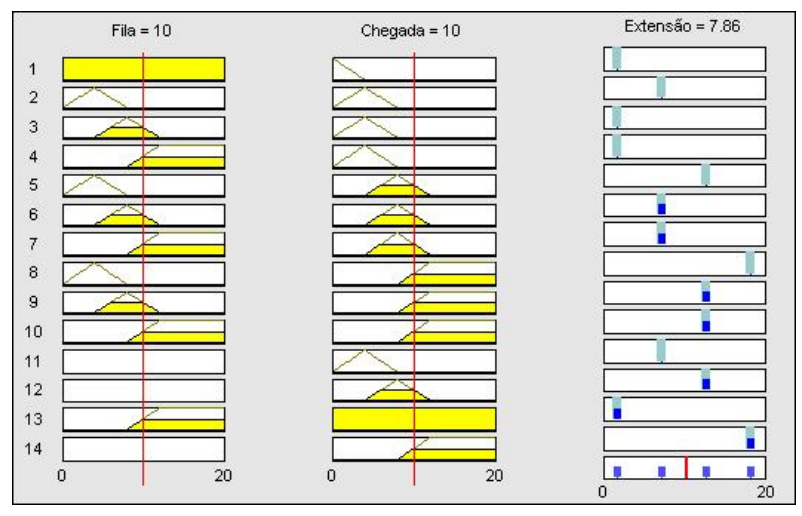

(a) Regras

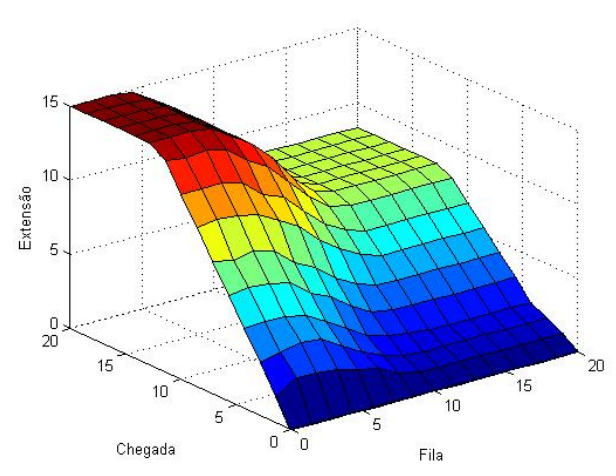

(b) Superfície de controle

Figura 5: Base de regras e superfície de resposta do Controlador 4 
Foram realizadas 10 simulações de 1 hora para cada um dos controladores testados e para cada nível de volume estudado (Tabela 2), totalizando 120 horas de simulação.

Tabela 2: Tabela 2: Volumes de tráfego simulados (dados em veículos/hora)

\begin{tabular}{lccc}
\hline Volume & Via principal & Via secundária & TOTAL \\
\hline Baixo & 600 & 200 & 800 \\
Médio & 1.200 & 400 & 1.600 \\
Alto & 1.800 & 600 & 2.400 \\
\hline
\end{tabular}

O controle semafórico estabelecido na simulação é bifásico para uma interseção isolada totalmente atuada sob o modo de controle fuzzy. Foram testados quatro controladores fuzzy, sendo dois do tipo Mamdani (Controladores 1 e 3) e dois do tipo Sugeno (Controladores 2 e 4).

Através da análise das superfícies de resposta dos Controladores 3 e 4, observa-se que o controlador do tipo Sugeno (com variável de saída singletons) tende a dar extensões menores que seu correspondente com saída do tipo fuzzy (controlador de Mamdani).

\section{RESULTADOS OBTIDOS}

As análises foram realizadas por pares de controladores: Controladores 1 e 2 (Tabela 3), e Controladores 3 e 4 (Tabela 4).

As diferenças percentuais dos valores médios dos resultados de simulação para os pares de controladores foram apresentadas no sentido de facilitar as análises comparativas. Para este fim, o controlador de Mamdani foi adotado como o elemento de referência para o estudo proposto (ver Equação 1).

$$
D P=\frac{V S_{i}-V M_{i}}{V M_{i}} \cdot 100
$$

onde, $D P$ : diferença percentual;

$V S_{i}$ : valor médio da variável $i$ fornecido pelo controlador Sugeno;

$V M_{i}$ : valor médio da variável $i$ fornecido pelo controlador de Mamdani.

Foram realizados, também, testes de hipótese de igualdade entre médias para a avaliação da diferença dos controladores testados em termos de medidas de desempenho do tráfego (atraso médio e número de paradas veiculares) e da resposta dos controladores (tempo médio de verde e comprimento médio de ciclo). Para tanto, foi adotado o nível de significância $\alpha=5 \%$. Para a realização dos testes, os resultados de simulação (10 horas para cada caso) podem ser considerados conjuntos de dados emparelhados para cada hora de simulação independente, uma vez que o simulador utilizado permite gravar um padrão de chegadas veiculares e reutilizá-lo para vários testes. Nas Tabe- las 3 e 4 os resultados dos testes realizados são apresentados em termos de "S", para indicar diferenças significativas, e "NS", caso contrário.

\subsection{Controladores 1 e 2}

O desempenho do tráfego, analisado através das variáveis "atraso médio" e "percentual de paradas veiculares", foi melhor sob o controle do tipo Sugeno, especialmente para os volumes alto e médio. Tal vantagem foi verificada tanto para a interseção como um todo, quanto para a as aproximações consideradas individualmente (via principal e via secundária). Destes resultados, apenas em um caso (paradas veiculares para a via secundária sob volume alto) o controlador de Mamdani apresentou resultados melhores, sendo que a diferença observada para o controlador de Sugeno, para este caso, foi não significativa sob o teste de hipótese realizado (Tabela 3). Para o volume baixo os resultados mostram que o controlador de Sugeno tende a beneficiar a via secundária, trazendo prejuízo para a via principal, e conseqüentemente, para a interseção.

Tabela 3: Diferença percentual entre os resultados dos controladores 1 e 2, e significância segundo o teste de Hipótese

\begin{tabular}{|c|c|c|c|c|}
\hline \multicolumn{2}{|c|}{ Controladores 1 e 2} & \multicolumn{3}{|c|}{ Diferença Percentual (DP) } \\
\hline Variável & Aproximação & V. Alto & V. Médio & V. Baixo \\
\hline \multirow{6}{*}{$\begin{array}{l}\text { ATRASO } \\
\text { MÉDIO } \\
\text { (s) }\end{array}$} & \multirow{2}{*}{ Interseção } & -10.7 & -4.4 & 2.3 \\
\hline & & $\mathrm{S}$ & $\mathrm{S}$ & NS \\
\hline & Via & -12.0 & -1.7 & 8.9 \\
\hline & Principal & $\mathrm{S}$ & NS & $\mathrm{S}$ \\
\hline & Via & -8.5 & -9.3 & -14.2 \\
\hline & Secundária & $\mathrm{S}$ & $\mathrm{S}$ & $\mathrm{S}$ \\
\hline \multirow{6}{*}{$\begin{array}{l}\text { PARADAS } \\
\text { VEICULARES } \\
(\%)\end{array}$} & \multirow{2}{*}{ Interseção } & -8.0 & -3.0 & 3.7 \\
\hline & & $\mathrm{S}$ & NS & S \\
\hline & Via & -12.9 & -2.5 & 10.9 \\
\hline & Principal & $S$ & NS & $\mathrm{S}$ \\
\hline & Via & 0.4 & -4.0 & -12.1 \\
\hline & Secundária & NS & NS & $\mathrm{S}$ \\
\hline \multirow{4}{*}{$\begin{array}{l}\text { VERDE } \\
\text { MÉDIO } \\
\text { (s) }\end{array}$} & Via & -18.5 & -21.3 & -19.2 \\
\hline & Principal & $\mathrm{S}$ & $\mathrm{S}$ & $\mathrm{S}$ \\
\hline & Via & -25.9 & -16.3 & -3.6 \\
\hline & Secundária & $S$ & $S$ & S \\
\hline \multirow{2}{*}{$\begin{array}{r}\text { COMPRIMENTO } \\
\text { DE CICLO (s) }\end{array}$} & \multirow{2}{*}{ Interseção } & -17.0 & -14.4 & -8.1 \\
\hline & & $S$ & $S$ & $S$ \\
\hline
\end{tabular}

S: diferença significativa; NS: diferença não-significativa

$\mathrm{Na}$ análise da resposta do controlador, os resultados apresentaram diferenças significativas, para um nível de significância de $5 \%$. Pode ser observado que tanto os tempos médios de verde quando o ciclo médio foram maiores para os controladores do tipo Mamdani. Sendo assim, fica evidente que o tipo de controlador utilizado impacta significativamente na resposta do controlador (tempo de verde e tempo de ciclo).

\subsection{Controladores 3 e 4}

Ao avaliar as medidas de desempenho do tráfego, por meio das diferenças percentuais de atraso médio e de 
paradas veiculares para esse par de controladores, observou-se que o controlador de Sugeno (Controlador 3) apresentou melhores resultados para a maioria das situações avaliadas, com apenas dois resultados contrários, sendo que um deles foi não significativo (Tabela 4).

Tabela 4: Diferença percentual entre os resultados dos controladores 3 e 4, e significância segundo o teste de Hipótese

\begin{tabular}{|c|c|c|c|c|}
\hline \multicolumn{2}{|c|}{ Controladores 3 e 4} & \multicolumn{3}{|c|}{ Diferença Percentual (DP) } \\
\hline Variável & Aproximação & V. Alto & V. Médio & V. Baixo \\
\hline \multirow{6}{*}{$\begin{array}{l}\text { ATRASO } \\
\text { MÉDIO } \\
\text { (s) }\end{array}$} & \multirow{2}{*}{ Interseção } & -23.2 & -20.2 & -9.1 \\
\hline & & $\mathrm{S}$ & $\mathrm{S}$ & $\mathrm{S}$ \\
\hline & \multirow{2}{*}{$\begin{array}{c}\text { Via } \\
\text { Principal }\end{array}$} & -28.7 & -22.7 & -1.1 \\
\hline & & $\mathrm{S}$ & $\mathrm{S}$ & NS \\
\hline & \multirow{2}{*}{$\begin{array}{c}\text { Via } \\
\text { Secundária }\end{array}$} & -11.8 & -15.1 & -24.4 \\
\hline & & $\mathrm{S}$ & $\mathrm{S}$ & $\mathrm{S}$ \\
\hline \multirow{6}{*}{$\begin{array}{r}\text { PARADAS } \\
\text { VEICULARES } \\
(\%)\end{array}$} & \multirow{2}{*}{ Interseção } & -15.6 & -13.9 & -2.1 \\
\hline & & $\mathrm{S}$ & $\mathrm{S}$ & NS \\
\hline & \multirow{2}{*}{$\begin{array}{c}\text { Via } \\
\text { Principal }\end{array}$} & -24.6 & -20.3 & 3.8 \\
\hline & & S & $\mathrm{S}$ & NS \\
\hline & \multirow{2}{*}{$\begin{array}{c}\text { Via } \\
\text { Secundária }\end{array}$} & 2.9 & -2.1 & -12.8 \\
\hline & & $\mathrm{S} *$ & NS & $\mathrm{S}$ \\
\hline \multirow{4}{*}{$\begin{array}{l}\text { VERDE } \\
\text { MÉDIO } \\
\text { (s) }\end{array}$} & \multirow{2}{*}{$\begin{array}{c}\text { Via } \\
\text { Principal }\end{array}$} & -20.9 & -33.7 & -41.9 \\
\hline & & $S$ & $\mathrm{~S}$ & $\mathrm{~S}$ \\
\hline & \multirow{2}{*}{$\begin{array}{c}\text { Via } \\
\text { Secundária }\end{array}$} & -39.2 & -42.9 & -27.9 \\
\hline & & $\mathrm{S}$ & $\mathrm{S}$ & S \\
\hline \multirow{2}{*}{$\begin{array}{r}\text { COMPRIMENTO } \\
\text { DE CICLO (s) }\end{array}$} & \multirow{2}{*}{ Interseção } & -23.8 & -30.5 & -27.1 \\
\hline & & $\mathrm{S}$ & $\mathrm{S}$ & $\mathrm{S}$ \\
\hline
\end{tabular}

S: diferença significativa; NS: diferença não-significativa *: caso significativo desfavorável ao controlador Sugeno

As diferenças relativas à resposta dos controladores (verde médio e comprimento de ciclo) foram todas significativas, e em todos os resultados as medidas do controlador de Sugeno foi menor. Novamente, comprova-se a influência do tipo de controlador utilizado na resposta de controle, bem como no desempenho do tráfego.

Os resultados obtidos (Tabelas 3 e 4) permitem ainda concluir que o desempenho do tráfego é, também, afetado pela interação entre o tipo de controlador utilizado e os volumes a serem controlados. Através da realização de uma Análise de Variância, considerando os fatores "Controlador" e "Volume", ficou efetivamente comprovada a interação entre os fatores ao nível de significância de 5\%, exceto para o atraso médio na via secundária.

\section{CONCLUSÕES}

Diante dos resultados obtidos neste estudo é possível observar que diferentes tipos de controladores impactam de modo significativo tanto na resposta do controlador, como também no desempenho do tráfego. Sendo assim, é de suma relevância a atenção do projetista na construção do controlador fuzzy, dentre os diversos aspectos já apresentados na literatura, para a definição do tipo de controlador a ser utilizado - se do tipo
Mamdani ou Sugeno.

Para as condições de tráfego analisadas (volumes e características gerais da interseção considerada) o controlador do tipo Sugeno apresentou melhores resultados sob o desempenho do tráfego, bem como menores tempo de verde e de ciclo, para os volumes analisados. No entanto, como existe a interação entre tipo de controlador e volume, a decisão pela escolha do controlador tipo Sugeno deve ser precedida de uma avaliação preliminar para verificar se, para o caso considerado esse tipo de controlador é, efetivamente, o mais apropriado. Para tanto, um estudo nos moldes do realizado no presente artigo é recomendado.

Vale ressaltar que as vantagens dos controladores do tipo Sugeno sobre os do tipo Mamdani observadas nesse estudo devem ser somadas às vantagens de concepção destes controladores, uma vez que possuem menos elementos a serem definidos. A função de implicação e os métodos de defuzificação são elementos dispensáveis na concepção dos controladores do tipo Sugeno e, como pode ser observado na literatura (Jacques et al, 2002b e 2002c), impactam significativamente no processo do controle realizado.

É importante dar continuidade aos estudos relacionados à comparação dos controladores de Mamdani e Sugeno avaliando condições geométricas e operacionais diferentes das consideradas nesse estudo. Variações dos volumes médios de tráfego, movimentos de conversão e a operação em rede devem ser considerados em estudos futuros.

\section{REFERÊNCIAS BIBLIOGRÁFICAS}

Andrade, M.; Jacques, M. A. P. (2005) Estudo para aprimoramento da operação de controladores semafóricos fuzzy. In: XIX ANPETCongresso de Pesquisa e Ensino em Transportes, 2005, Recife. Rio de Janeiro: ANPET, 2005. v. 1. p. 817-828.

Bingham, E. (1998) Neurofuzzy Traffic Signal Control. Department of Engineering Physics and Mathematics. Helsinki Univerity of Tecnology.

Jacques, M.A.P., J. Niittymäki, and M. Pursula (2002a) Analysing Different Fuzzy Traffic Signal Controllers for Isolated Intersections. Paper accepted for presentation at TRB 81st Annual Meeting. January 13-17, 2002, Washington, DC.

Jacques, M. A. P.; J. Nittymäki; M. Pursula, e I. Kosonen (2002b) The impact of different approximate reasoning methods on fuzzy signal controllers. Anais do 13th Mini-Euroconference, 10 a 13 de Junho de 2002, Bari - Itália, p.184 - 192.

Jacques, M. A. P.; J. Nittymäki; M. Pursula, e I. Kosonen (2002c) Analysis of Different Defuzzyfication Methods Applied to Fuzzy Signal Controller. In: XII Congresso Panamericano de Ingeniería de Tránsito y Transport. Quito - Equador.

Jacques, M. A. P.; D. B. L. M. Santos, M. Pursula e I. Kosonen (2003) Studying the Importance of Fuzzy Sets Definitions for Fuzzy Signal Controllers. In: Fourth International Symposium on Uncertainty Modeling and Analysis - INSUMA 2003, Maryland, USA, p. $64-69$.

Kikuchi, S. (1991) Aplication of fuzzy set theory to the analysis of transportation problems. In: Second International Conference on Applications of Advanced Technologies in Transportation Enginnering. Minneapolis, Minnesota, August 18-21, 1991.

Kosonen, I. (1999) HUTSIM - Urban Traffic Simulation and Control Model: Principles and Applications. Publication 100, Helsinki 
University of Technology, Transportation Engineering, Espoo, Finland, 1999, $248 \mathrm{p}$

Lee, C. C. (1990a) Fuzzy Logic in Control Systems: Fuzzy Logic Controller, Part I. IEEE Transactions on Systems, Man, and Cybernetics, Vol. 20, No. 2, March/April 1990, pp. $404-418$.

Lee, C. C. (1990b) Fuzzy Logic in Control Systems: Fuzzy Logic Controller, Part II. IEEE Transactions on Systems, Man, and Cybernetics, Vol. 20, No. 2, March/April 1990, pp. 419 - 435.

Mamdani, E. H. (1973) Aplications of fuzzy algorithms for control of simple dynamic plant. Proc. IEEE 121, vol. 12, p. 1585-1588.

Mamdani, E. H. e Assilian S. (1975) An experiment in linguistic synthesis with a fuzzy logic controller. Int. J. Man-Machine Studies, vol. 7, p. 1-13.

MathWorks (2008) Getting Started Guide. Matlab ${ }^{\circledR 7}$.

Niittymäki, J. and M. Pursula (2000) Signal Control Using Fuzzy Logic. Fuzzy Sets and Systems, Vol. 116, pp.11-22.

Niittymäki, J. and R. Nevala (2001) Fuzzy Adaptive Traffic Signal Control - Principles and Results. In: Joint 9th IFSA World Congress and 20th NAFIPS International Conference, Vancouver, Canada, July 2001, 6 p.

Pappis, C. P.; H. Mamdani (1977) A Fuzzy Logic Controller for Traffic Junctions. IEEE Transactions on Systems, Man and Cybernetics. Vol. SMC-7, No 10.

Santos, D, B, (2003) Procedimento para a Construção dos Conjuntos Fuzzy Utilizados em Controladores Semafóricos, Dissertação de Mestrado,Universidade de Brasília, Brasília, DF.

Sugeno, M. (1985). An introductory survey of fuzzy control. Information Sciences 36, p. 59-83.

Takagi, T. e Sugeno M. (1983) Derivation of fuzzy control rules from human operator's control action. IFAC Symposium on Fuzzy Information, Knowledge Representation and Decision Analysis, Marseille, p. 55-60.

Vaz, A M (2006) Estudos das Funções de pertinência para conjuntos fuzzy utilizados em Controladores Semafóricos Fuzzy. Dissertação de Mestrado, Departamento de Engenharia Civil e Ambiental, Faculdade de Tecnologia Universidade de Brasília, DF, 158 p.

Zadeh, L. A. (1973) Outline of a new approach to the analysis of complex systems and decision processes. IEEE Transactions on Systems, Man, and Cybernetics, Vol. SMC-3, No. 1, p. 28-44.

Zimmermann, H.-J. (1996) Fuzzy Set Theory and Its Applications. 3rd Edition. Kluwer Academic Publishers. 